\title{
Carbon nanotube nanocomposite-modified paper electrodes for supercapacitor applications
}

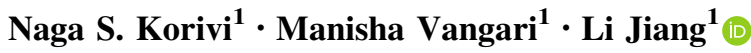

Received: 18 August 2016/Accepted: 19 December 2016/Published online: 27 December 2016

(c) The Author(s) 2016. This article is published with open access at Springerlink.com

\begin{abstract}
This paper describes the evaluation of carbon paper electrodes for supercapacitor applications. The electrodes are based on carbon micro-fiber paper modified with active material consisting of layers of silver nanoparticulate ink and a nanocomposite of multi-walled carbon nanotubes and silver nano-particulate ink. The electrodes were characterized microscopically and electrically. Current-voltage studies revealed a consistent Ohmic behavior of the electrode when modified with different nanostructured active material. Among the active materials incorporated into the electrode, a nanocomposite of carbon nanotubes and silver nano-particulate ink significantly improved capacitance. The paper electrodes can be used for lightweight and ultrathin supercapacitors and other portable energy applications.
\end{abstract}

Keywords Carbon supercapacitors - Supercapacitor · Paper supercapacitor · Carbon nanotubes

\section{Introduction}

The recent decades have witnessed growing interest in energy storage devices for portable applications. Among the energy devices being developed, the supercapacitor holds significant promise for portable and other macrolevel applications requiring relatively large electric charge. A supercapacitor is similar to a capacitor with one exception in that it exhibits much larger capacitance than a

Li Jiang

ljiang@mytu.tuskegee.edu

1 Department of Electrical Engineering, Tuskegee University, Tuskegee, AL 36088, USA typical capacitor. This is made possible by different mechanisms, primarily including the mode of operation, the device structure and constituent materials. For portable applications, the typical requirements involve high energy density, lightweight structure and in some cases structural flexibility. Thin and lightweight film type device structures potentially allow for these criteria. This is the primary reason why there has been recent research interest in thin film and flexible supercapacitors. One of the approaches to make these types of devices is using paper and fabric in their structure, primarily as the basic electrode materials. Recent developments on this front have focused on rendering paper and fabric substrates suitable for electrode applications. This has primarily involved the use of carbon nanotubes (CNTs) to make the substrates electrically conductive while increasing their surface area significantly, among other reasons. Some reports have employed relatively complex methods and high-temperature processes to deposit CNTs on paper and fabric substrates (Yuan et al. 2012; Hu et al. 2010; Kim et al. 2012). To address such technology constraints, we developed relatively easy and room-temperature processes to incorporate nanostructured active material including CNTs on carbon micro-fiber paper surfaces (Korivi et al. 2012). These processes based on established thin and thick film technology allowed us to fabricate novel supercapacitor electrodes that were thin and lightweight.

In this paper, we report on the development and investigation of supercapacitor electrodes made of carbon paper surfaces modified by active nanostructured materials. We present observations from our evaluation of different active materials. This evaluation is primarily focused on studying the electrodes microscopically and electrically. The electrical characterization includes studies on current-voltage behavior of the electrodes when coated with a particular

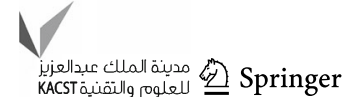


active material and possible implications on supercapacitor applications. This particular aspect has not been adequately addressed by contemporary research on the development of paper-based supercapacitors.

\section{Experimental}

\section{Materials}

The electrodes investigated in this work were fabricated on commercially available carbon micro-fiber paper. Two different thickness variants of this paper, namely Toray TGP-H-030 $(100 \mu \mathrm{m})$ and Toray TGP-H-060 $(190 \mu \mathrm{m})$ were obtained from Fuel Cell Store, USA and used as the base electrode material. The active material was primarily based on two materials-multi-walled carbon nanotubes (MWCNTs) (Product \#659258, Sigma-Aldrich Co. LLC, USA) and commercially available silver (Ag) nano-particulate ink (UT DOTs, Inc., USA). The Ag nano-particulate ink is referred to as $\mathrm{Ag}$ nano-ink in this paper. The $\mathrm{Ag}$ nano-ink was either used in its procured form or solution blended with MWCNTs ( $1 \mathrm{ml}$ of $\mathrm{Ag}$ nano-ink $+50 \mathrm{mg}$ MWCNTs) to form a nanocomposite (Ag nano-ink/ MWCNTs). The electrolyte material was based on a composite of polyvinyl alcohol (PVA) and phosphoric acid $\left(\mathrm{H}_{3} \mathrm{PO}_{4}\right)$. PVA (Product \#348406, MW 13,000-23,000, Sigma-Aldrich Co. LLC, USA) and phosphoric acid (Product \#438081, $\geq 85$ wt $\%$ in $\mathrm{H}_{2} \mathrm{O}$, Sigma-Aldrich Co. LLC) were used for this purpose.

\section{Fabrication}

The solid electrolyte was fabricated by solution casting a mixture of PVA and $\mathrm{H}_{3} \mathrm{PO}_{4}$. The first step was preparing a solution of PVA and $\mathrm{H}_{3} \mathrm{PO}_{4}$. PVA ( $3 \mathrm{~g}$ ) was dissolved in $20 \mathrm{ml}$ of deionized (DI) water at $65{ }^{\circ} \mathrm{C}$ with magnetic stirring. To this solution, $2 \mathrm{ml}$ of $\mathrm{H}_{3} \mathrm{PO}_{4}$ was added and the mixture was thoroughly blended by magnetic stirring for 5 min. Finally, the mixed solution was poured on a plastic surface and allowed to cure (solidify) at room temperature $\left(\sim 35^{\circ} \mathrm{C}\right.$ ) overnight. Subsequently, the solid PVA/ $\mathrm{H}_{3} \mathrm{PO}_{4}$ layer was manually detached from the underlying plastic surface, to yield a free-standing solid electrolyte layer of approximately $200 \mu \mathrm{m}$ thickness. Based on active material, three types of electrodes were investigated, namelyelectrode \#1 (plain carbon paper), electrode \#2 (carbon paper $+\mathrm{Ag}$ nano-ink $+\mathrm{Ag}$ nano-ink/MWCNTs), and electrode \#3 (carbon paper $+\mathrm{Ag}$ nano-ink $+\mathrm{Ag}$ nanoink/MWCNTs + MWCNTs). All three electrodes had typical lateral dimensions of $1 \mathrm{~cm} \times 1 \mathrm{~cm}$. Electrode \#1 was a pure carbon paper without active material (Fig. 1a). Electrode \#2 was fabricated by sequentially spin-coating a b
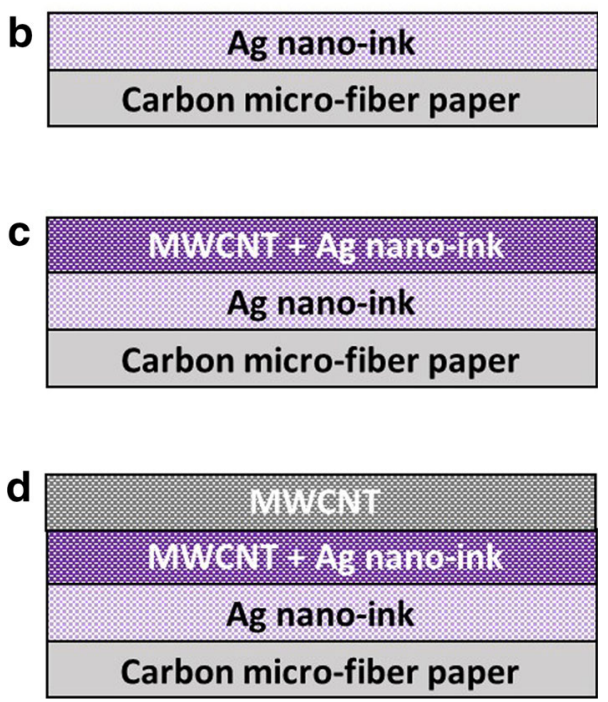

Fig. 1 Schematic of electrode fabrication. The figure is not to scale. Created by Microsoft Office PowerPoint

layer of Ag nano-ink on the carbon paper surface, followed by a composite layer of $\mathrm{Ag}$ nano-ink and MWCNTs (Fig. 1b, c). The spin-coating involved spinning the carbon paper horizontally at $150 \mathrm{rpm}$ for $50 \mathrm{~s}$ and dispensing droplets of active material $(\sim 0.2-0.3 \mathrm{ml})$ on the spinning surface. This was done to localize active material on the surface, rather than allow it to seep through the paper. This process varies from a typical spin-coating process which involves dispensing liquid material on a surface prior to spinning the surface. Electrode \#3 was similar to electrode \#2 with one additional layer of pure MWCNTs (Fig. 1d). This layer was formed by casting a solution of MWCNTs in acetone.

\section{Electrode characterization}

The electrodes fabricated in this work were characterized microscopically by scanning electron microscopy and electrically by current-voltage (I-V) testing. The latter was done using a Keithley 4200 SCS semiconductor characterization system. To conduct the I-V tests on the electrodes, a clean glass surface was coated with conducting silver paint and an electrode was placed on the silver (Fig. 2a). All the I-V tests were done at room temperature $\left(\sim 35^{\circ} \mathrm{C}\right)$. The three different electrodes were investigated for supercapacitor applications. This was done by sandwiching the solid electrolyte between two electrodes of the same type, and holding all the layers together in a mechanical vise. An example is shown in the case of electrode \#3 (Fig. 2b). Subsequently, the device was 
Fig. 2 Schematic of a currentvoltage measurements on the electrodes; $\mathbf{b}$ assembly of electrodes and electrolyte in a mechanical vise to form a supercapacitor device and its capacitance testing. The figure is not to scale. Created by Microsoft Office PowerPoint

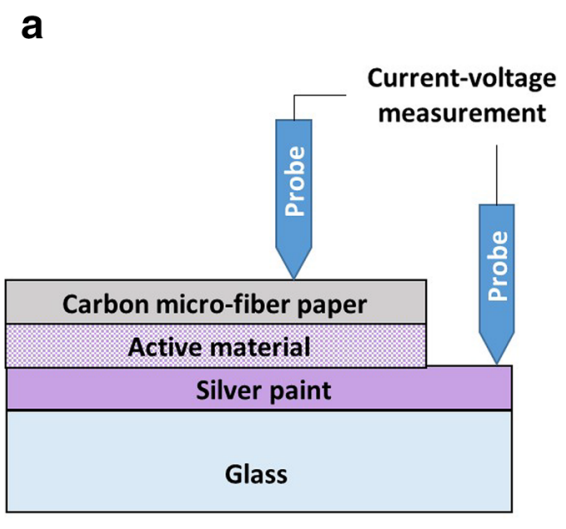

measured for its capacitance using an HP 4284A Precision LCR meter (Fig. 2b).

\section{Results and discussion}

Scanning electron microscopy (SEM) was used to microscopically study the carbon paper substrate and the distribution of active material over the paper surfaces (TGP-H060). SEM images of an electrode \#3 surface are shown in
Fig. 3. A corner of the electrode surface is shown in Fig. 3a. As highlighted in the figure, the dark rod-like structures are the micro-fibers of the pure carbon paper substrate. The brighter material at the bottom left corner of the image is some electrode material coated on the substrate. Figure $3 \mathrm{~b}$ shows an image taken by tilting the electrode, showing a perspective profile. We can clearly see the active material (highlighted) deposited on the underlying micro-fibers of the carbon paper substrate. The coverage of active material is not uniform in this particular
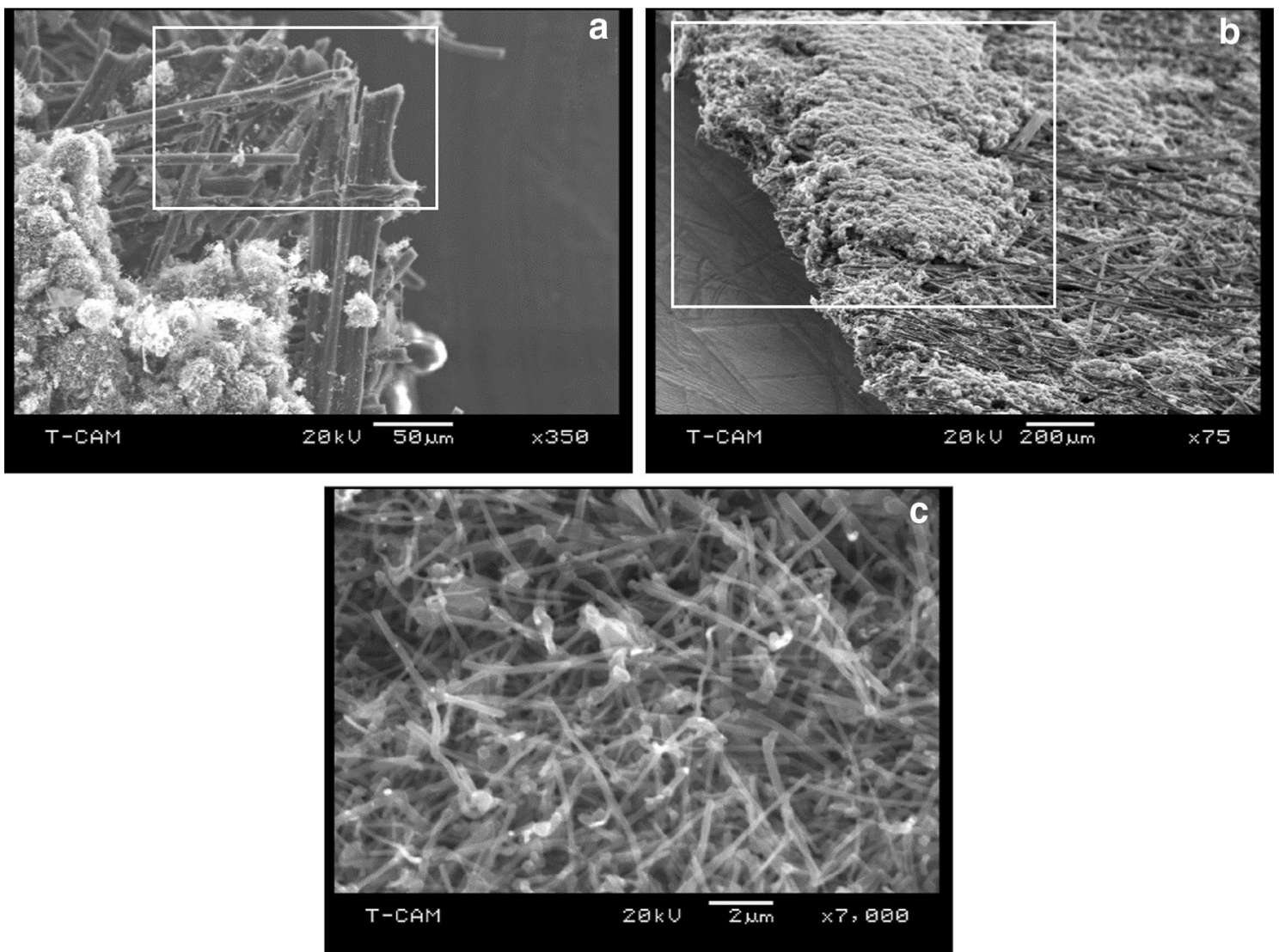

Fig. 3 SEM micrographs showing a the micro-fiber structure of the carbon paper substrate (highlighted); b active material (highlighted) deposited on the carbon paper; c magnified image of electrode surface, showing a network of MWCNTs

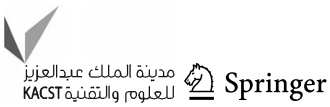


case due to its partial removal during handling for the SEM imaging. Overall, the SEM images also show that the micro-fiber network of the carbon paper substrate is penetrated by the active material. Magnified imaging of the electrode surface shows that it is covered by a dense network of MWCNTs (Fig. 3c). The current-voltage (I-V) behavior of the different electrodes was studied in this work. Figure 4 presents the I-V plots of the three different electrodes made on TGP-H-030 paper. As can be seen in this figure, the I-V characteristics of the three different electrodes are Ohmic in nature. This is important and desirable when considering electrical contacts. In addition, the I-V curves of the three electrodes are not significantly different from each other, with a slight improved currentvoltage behavior shown by electrode \#3 compared to electrodes \#1 and \#2.

Electrode \#2 shows slightly improved I-V behavior compared to electrode \#1. An important observation here is that an Ohmic behavior is retained by the carbon paper even after the incorporation of active material such as Ag nano-ink and MWCNTs. This is again a desirable quality for the use of such electrodes in supercapacitor applications. In Fig. 4, the current for all three electrodes shows saturation at $0.1 \mathrm{~A}$. This is because of a test limit of $0.1 \mathrm{~A}$ set on the testing equipment prior to the measurements.

The three electrodes were evaluated in supercapacitor device configuration. It was observed that the supercapacitor device made by electrode \#3 showed higher areal

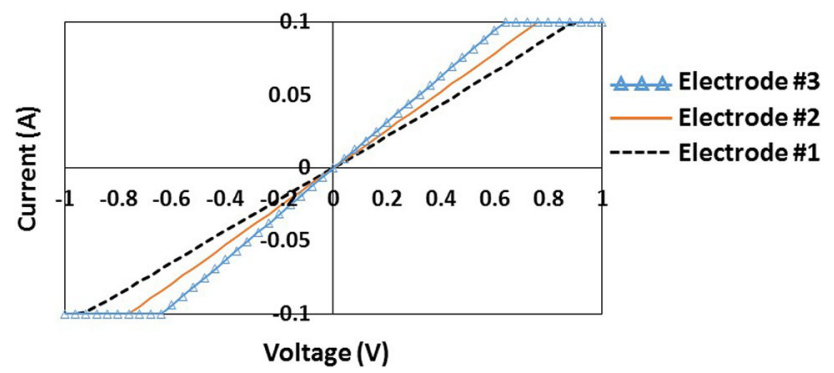

Fig. 4 Current-voltage plots of electrodes evaluated in this work. Created by Microsoft Office Excel

Table 1 Electrical and other parameters measured from supercapacitors developed in this work

\begin{tabular}{|c|c|}
\hline Parameter & Value \\
\hline $\begin{array}{l}\text { Maximum areal } \\
\text { capacitance }\end{array}$ & $\begin{array}{l}\sim 2 \mathrm{mF} / \mathrm{cm}^{2} \text { (carbon paper }+ \text { Ag nano-ink }+ \\
\text { Ag nano-ink/MWCNTs }+ \text { MWCNTs) } \\
<0.1 \mathrm{mF} / \mathrm{cm}^{2} \text { (carbon paper }+ \text { Ag nano-ink } \\
\quad+\text { MWCNTs) }\end{array}$ \\
\hline Maximum voltage & $\sim 2 \mathrm{~V}$ \\
\hline Total thickness & $450-750 \mu \mathrm{m}$ \\
\hline Total weight & $\leq 0.5 \mathrm{mg}$ \\
\hline
\end{tabular}

capacitance than the supercapacitor made by electrodes \#2 and \#1. The device with electrode \#3 shows a maximum areal capacitance of approximately $2 \mathrm{mF} / \mathrm{cm}^{2}$ at $20 \mathrm{~Hz}$, an improvement over similar devices which show comparable electrical performance, but have larger total weight and thickness than our device (Hu et al. 2012). Devices with electrode \#3 can charge up to $2 \mathrm{~V}$. These qualities make this developed device an ideal candidate for portable energy applications. A major contributing factor to this device's superior electrical performance is electrode \#3's unique combination of nano-structured active materials. The fact that these active materials were deposited by simple processes is an improvement over other reports on similar devices which employ relatively complex processes to deposit active nano-material on paper substrates (Yuan et al. 2012; Hu et al. 2010). A summary of key results from our supercapacitor development is provided in Table 1.

Observations indicate that the incorporation of the layer of Ag nano-ink/MWCNT composite is significant in terms of the overall performance of the supercapacitor (Wee et al. 2010). In an earlier work, we evaluated electrodes based on the same type of carbon paper coated with a layer of silver nano-particulate ink, followed by a layer of MWCNTs (Korivi et al. 2012). These electrodes were used to make a supercapacitor which showed a maximum areal capacitance of $<0.1 \mathrm{mF} / \mathrm{cm}^{2}$ measured at a frequency of $20 \mathrm{~Hz}$. This is significantly lesser than the case of a device made by electrode \#3 which incorporates the Ag nano-ink/ MWCNT nanocomposite layer.

There are a few considerations that should be discussed. The electrical characteristics of the electrode can be improved by modifying the carbon nanotubes to improve their dispersion behavior. To illustrate, it is well established that using functionalized and treated CNTs improves their dispersion behavior and decreases agglomeration, potentially improving their electrical characteristics (Tang et al. 2010; Ramasubramaniam et al. 2003; Chou et al. 2008; Ma et al. 2010; Sahoo et al. 2010). Another avenue for improvement is in the fabrication procedure, more specifically, the optimization of the spin-coating process of depositing active materials on carbon paper substrates.

The choice of method to deposit active material is to an extent influenced by the type of material being deposited. The deposition of Ag nano-ink on a spinning paper substrate is a viable technique to deposit this type of active material. Previous experiments with solution casting of $\mathrm{Ag}$ nano-ink showed that the material seeped through the paper substrate and did not improve supercapacitor device performance. However, in the case of depositing MWCNTs, the solution casting method was a suitable method. Our experiments with a spin-coating process to deposit MWCNTs yielded electrodes which did not show appreciable improvement over electrodes with only an Ag nano- 
ink coating. The use of solution casting, a room-temperature method to deposit CNTs on the micro-fiber paper is an improvement over other reports which employed a higher temperature chemical vapor deposition for the same purpose.

A major advantage of using micro-fibrous paper as the basic electrode material is the inherent surface area available. One aspect of using micro-fibrous paper substrates such as that in the present work is that it is easy for liquid materials to seep through the entire thickness of the paper. This can pose problems while depositing active material on the paper. Our results show the feasibility of using spin coating to deposit active materials on paper substrates. In some cases, this spin-coating process can be viable alternate to other methods. At this juncture, the spin-coating process is not optimized. If the spin-coating speed (rpm) is too low, then there is a possibility of the active material seeping through the paper substrate. If the spin rpm is too high, then insufficient material gets deposited on the paper, with most of the material getting thrown off from the spinning paper substrate. It is, therefore, essential to optimize the spin speed for a particular active material.

\section{Conclusions}

Paper electrodes have been developed in this work by depositing nanostructured active material on carbon microfiber paper substrates. The electrodes were characterized microscopically and electrically. Current-voltage tests show that the developed electrodes exhibit Ohmic behavior with silver contacts. The electrodes were also evaluated in a supercapacitor configuration. The presence of the nanostructured active material clearly improves the capacitive behavior. Further improvements are possible with design and fabrication optimization. The currentvoltage studies and capacitance measurements indicate that the nanostructured materials and their combinations investigated in this work are feasible active material candidates on this type of carbon paper surfaces.

Acknowledgements The authors thank Mr. Melvin DeBerry for assistance with the current-voltage measurements. The authors thank Dr. Vijay Rangari and Mr. Chinedu Okoro of the Tuskegee University
Center for Advanced Materials (T-CAM) for Scanning Electron Microscopy (SEM) micrographs. This work was funded by the United States Army Research Office Contract \#W911NF-11-1-0214 and Department of Homeland Security Grant \# 2012-ST-062-000055.

Open Access This article is distributed under the terms of the Creative Commons Attribution 4.0 International License (http:// creativecommons.org/licenses/by/4.0/), which permits unrestricted use, distribution, and reproduction in any medium, provided you give appropriate credit to the original author(s) and the source, provide a link to the Creative Commons license, and indicate if changes were made.

\section{References}

Chou WJ et al (2008) Characteristics of polyimide-based nanocomposites containing plasma-modified multi-walled carbon nanotubes. Compos Sci Technol 68:2208-2213

$\mathrm{Hu}$ LB, Wu H, Cui Y (2010) Printed energy storage devices by integration of electrodes and separators into single sheets of paper. Appl Phys Lett 96(18). doi:10.1063/1.3425767 (Article ID: 183502)

Hu S, Rajamani R, Xu X (2012) Flexible solid-state paper based carbon nanotube supercapacitor. Appl Phys Lett 100(10). doi:10. 1063/1.3691948 (Article ID: 104103)

Kim B, Chung H, Kim W (2012) High-performance supercapacitors based on vertically aligned carbon nanotubes and nonaqueous electrolytes. Nanotechnology 23(15):155401. doi:10.1088/09574484/23/15/155401

Korivi NS, Vangari M, Mohammed D, Etzioni B, Henry K, Jiang L (2012) Thin film paper supercapacitor. In: Proc, 12th international workshop on micro and nanotechnology for power generation and energy conversion applications (PowerMEMS 2012), Atlanta, GA, pp 287-290

Ma PC et al (2010) Dispersion and functionalization of carbon nanotubes for polymer-based nanocomposites: a review. Compos A $41: 1345-1367$

Ramasubramaniam R et al (2003) Homogeneous carbon nanotube/ polymer composites for electrical applications. Appl Phys Lett 83:2928-2930

Sahoo NG et al (2010) Polymer nanocomposites based on functionalized carbon nanotubes. Prog Polym Sci 35:837-867

Tang QY et al (2010) Study of the dispersion and electrical properties of carbon nanotubes treated by surfactants in dimethylacetamide. J Nanosci Nanotechnol 10:4967-4974

Wee G et al (2010) Particle size effect of silver nanoparticles decorated single walled carbon nanotube electrode for supercapacitors. J Electrochem Soc 157:A179-A184

Yuan LY et al (2012) Flexible solid-state supercapacitors based on carbon nanoparticles $/ \mathrm{MnO}_{2}$ nanorods hybrid structure. ACS Nano 6:656-661 